\title{
DESENVOLVIMENTO DE SISTEMA ESTRUTURADO COM INTELIGÊNCIA ARTIFICIAL PARA APOIO NO DIAGNÓSTICO DE PATOLOGIAS OFTALMOLÓGICAS MAIS RELEVANTES
}

\section{DEVELOPMENT OFASTRUCTURED ARTIFICIALINTELLIGENCE SYSTEMTO SUPPORTTHEDIAGNOSISOFTHEMOSTRELEVANTOPHTHALMICDISEASES}

\author{
Victor Antonio Kuiava1, Eliseu Luiz Kuiava², Eduardo Ottobelli Chielle ${ }^{3}$, \\ Roger Syllos ${ }^{1,4}$
}

\begin{abstract}
RESUMO
Introdução: A inteligência artificial (IA) está revolucionando a área da saúde. $\mathrm{Na}$ oftalmologia, esta tecnologia pode possibilitar diagnósticos mais rápidos e precisos, impedindo a progressão das alterações na visão. Médicos e algoritimosalgoritmos podem ser mais eficientes quando trabalham juntos. Desenvolver um software de IA com alta especificidade e sensibilidade para apoio no diagnóstico de algumas patologias oftalmológicas.
\end{abstract}

Métodos: O software de deep learning foi construído através de redes neurais valendo de duas bases computacionais MobileNet e Inception. Para o treinamento do banco de dados foram utilizadas 2.520 imagens de glaucoma, retinopatia diabética, toxoplasmose ocular, papiledema, descolamento de retina e retina normal. Para a validação foi utilizado 428 imagens patológicas e normais para os cálculos de sensibilidade e de especificidade. Todas as imagens foram cedidas da Sociedade Americana de Especialistas da Retina.

Resultados: Os resultados de sensibilidade e especificidade foram no MobileNet de 91\% (IC 95\%, 89-92\%) e 98,5\% (IC 95\%, 98-99\%); no Inception, de 91,4\% (IC 95\%, $89-93,5 \%$ ) de 98,4\% (IC 95\%, 98-98,8\%), respectivamente. Não houve diferença significativa entre os dois métodos utilizados.

Conclusão: O software apresentou resultados promissores na distinção das condições oftalmológicas pesquisadas.

Palavras-chave: Doenças oftálmicas; inteligência artificial; visão; oftalmologia

\section{ABSTRACT}

Introduction: Artificial intelligence (Al) is revolutionizing health care. In ophthalmology, this technology can enable faster and more accurate diagnoses, preventing the progression of vision Physicians and algorithms are most effective when working together. To develop an Al software with high specificity and sensitivity to support the diagnosis of some ophthalmic diseases.

Methods: A deep learning software was built through neural networks using two computational bases, MobileNet and Inception. For training the database, 2520 images of glaucoma, diabetic retinopathy, ocular toxoplasmosis, papilledema, retinal detachment, and normal retina were used. For validation, 428 pathological and normal images were used for calculations of sensitivity and specificity. All images were obtained from the American Society of Retina Specialists.

Results: The results of sensitivity and specificity were $91 \%$ (95\% confidence interval [Cl], 89-92\%) and 98.5\% (95\% Cl, 98-99\%) on MobileNet, and $91.4 \%(95 \% \mathrm{Cl}$, $89-93.5 \%)$ and $98.4 \%(95 \% \mathrm{Cl}, 98-98.8 \%)$ on Inception, respectively. There was no significant difference between the two methods.

Conclusion: The software showed promising results in distinguishing the main ophthalmic conditions surveyed.

Keywords: Ophthalmic diseases; artificial intelligence; vision; ophthalmology
Clin Biomed Res. 2021;41(1):27-32

1 Faculdade de Medicina, Universidade de Passo Fundo, Passo Fundo, RS, Brasil.

2 Departamento de Engenharia Elétrica, Universidade Internacional, São Miguel do Oeste, SC, Brasil.

3 Departamento de Ciências da Vida e Saúde, Universidade do Oeste de Santa Catarina. São Miguel do Oeste, SC, Brasil.

4 Departamento de Oftalmologia, Hospital São Vicente de Paulo, Passo Fundo, RS, Brasil.

Autor correspondente: Victor Antonio Kuiava victorkuiava@gmail.com Faculdade de Medicina, Universidade de Medicina Rua Teixeira Soares, 817 99010-080, Passo Fundo, Rio Grande do Sul, Brasil. 


\section{INTRODUÇÃO}

A Inteligência Artificial (IA) é uma área da ciência da computação que desponta como evolução na área da saúde e é parte integrante do avanço tecnológico que tem permitido sistemas de diferentes campos de atuação detectarem diversos problemas e tomarem decisões com base em grandes bancos de dados. A IA é capaz de imitar a habilidade de raciocínio humano, com as suas principais características, como aprendizagem, criatividade e habilidade de resposta, no entanto, é mais veloz, autônoma e com ausência de viesses humanos. Apresentam grande potencial para aprimorar funções desempenhadas exclusivamente por humanos, com destaque na área médica ${ }^{1-3}$.

Os exames complementares em especial de imagem também começam a ser analisados pela IA. Dentre as áreas médicas com grande potencial para serem aprimoradas, podemos citar a radiologia e a patologia. Essas duas especialidades compartilham em comum a necessidade de reconhecimento de padrões visuais para o diagnóstico adequado, papel que o computadores já são extremamente eficiente em outras áreas não médicas como reconhecimento facial e objetos ${ }^{4-7}$.

Nesta perspectiva, a oftalmologia apresenta o mesmo potencial, pois se utiliza de registros de imagem para diagnóstico através de exames de retinografia panorâmica, tomografia de coerência óptica e angiografia fluoresceínica digital. Existem vários trabalhos para estratificação de patologias oftalmológicas por computadores principalmente para retinopatia diabética ${ }^{3,8,9}$.

Em 2018, a FDA (Food and Drug Administration) aprovou o primeiro sistema de IA que realiza o diagnóstico de alterações oculares causadas por diabetes $^{9}$. A ferramenta de avaliação oftalmológica IDx-DR foi desenvolvida, testada e aprovada por uma empresa norte-americana de diagnósticos e utiliza redes neurais para detectar a retinopatia diabética por meio da análise dos olhos dos pacientes ${ }^{4}$. O Google desenvolveu uma IA para identificar retinopatia diabética e edema macular diabético, através da análise de fotos com sinais dessas doenças e o programa foi testado recentemente em pacientes do Hospital Aravind Eye (Índia), com resultados promissores ${ }^{10}$. No Brasil, já foi desenvolvida uma plataforma (redcheck) que ajuda na detecção de alterações, bem como auxilia o oftalmologista na confecção de laudos mais rápidos ${ }^{11}$.

As doenças oftalmológicas primárias bem como complicações oculares de doenças sistêmicas são condições relativamente frequentes e podem apresentar alta morbidades quando não devidamente tratadas, por acometer a visão sentido humano fundamental. Num contexto mundial, por características próprias da especialidade, existe uma dificuldade em conduzir diagnóstico e tratamento de doenças oftalmológicas ${ }^{12,13}$. Somado a outras circunstâncias como a falta de organização estrutural das redes de atenção básica, foi criado a Política Nacional de Atenção em Oftalmologia, para estimular o aprimoramento dos profissionais da saúde e na estruturação de fluxos de atendimento na atenção básica e no atendimento especializado ${ }^{14}$.

Neste sentido, a IA é uma importante aliada para a área de oftalmologia, pois contribui para a precisão dos diagnóstico. Portanto, o objetivo desse trabalho foi desenvolver um software estruturado em inteligência artificial para diagnosticar as principais patologias oftalmológicas que envolvem a retina e o nervo optico. com maior prevalência e potencial de danos irreversíveis a visão e que possa ser utilizado como uma ferramenta de triagem de patologias oftalmológicas em diversos níveis de atenção à saúde.

\section{MÉTODOS}

\section{Construção do software}

Construiu-se um programa computacional com interface de PHP versão 7 e Javascript. A fundamentação operacional utilização de inteligência artificial em deep learning, com duas opções de análise de inteligência artificial com bibliotecas disponibilizadas o Inception V3 e Mobilenet. Este software foi estruturado em um conjunto de algoritmos sobrepostos em diversas camadas de processamento, com várias transformações lineares e não lineares. Estabeleceu-se uma interface de comunicação computacional para a avaliação de radiografias simples e panorâmica.

O software desenvolvido foi denominado de EYE. A resolução de imagens do sistema foi de $224 \times 224$ pixeis para o MobileNet, 299x299 para o Inception sendo utilizado a análise de imagens com três portais $\mathrm{R}, \mathrm{G}$ e $\mathrm{B}$.

\section{Construção do banco de dados e seleção das lâminas histopatológicas}

O banco de dados foi constituído através de uma biblioteca open source para o aprendizado em TensorFlow. Dois modelos pré-treinados, MobileNet versão 1.0 e o Inception versão 3 foram utilizados, junto com um banco de dados de retinografias da American Society of Retina Specialists. Esse banco de dados foi constituído de um conjunto de imagens pré analisadas disponibilizadas pela Google Inc.

O projeto Retina Image Bank é um projeto da Sociedade Americana de Especialistas da 
Retina que disponibiliza um atlas de imagens préconferidas por 2 oftalmologistas especialistas em retina e vítreo. O projeto disponibiliza a utilização do banco de dados para pesquisas científicas que não tenham a intenção de fins lucrativos - categoria que enquadra este artigo.

Imagens de retinografias foram retiradas do site imagebank.asrs.org, de diversas categorias patológicas e normais. As condições analisadas foram retinopatia diabética (CID10 H36.0), tanto proliferativa, não proliferativa e edema macular; glaucoma $(\mathrm{H} 40)$, papiledema $(\mathrm{H} 47.1)$, toxoplasmose ocular (B58.0), descolamento de retina $(\mathrm{H} 33)$ e retina normal. Num total de 2.948 imagens foram utilizadas na elaboração do programa, sendo 2.520 para criar o banco de dados do treinamento e 428 imagens testas para a validação do programa. Todas essas imagens foram reavaliadas. As imagens selecionadas foram analisadas pelo software de inteligência artificial, criando o banco de dados.

Os resultados preliminares de confiabilidade do programa foram de 93\% para o MobileNet e 93,7\% para o Inception.

\section{Validação do software}

Após o software ter sido treinado, ele foi submetido a teste de eficiência que se deu em 3 etapas:

Primeiro: Foram selecionadas 737 retinografias patologias e normais, destas passaram por rotações de 90, 180 e 270 grau para aumentar o banco de dados. Assim 2.948 imagens foram conseguidas para o projeto.

Segundo: Foram criados dois grupos com o total de imagens, 428 imagens foram utilizadas como teste e validação do sistema e 2.520 foram usadas para construir o banco de dados do programa.

Terceiro: As imagens em formato Jpeg foram inseridas e analisadas no Software EYE, havendo a liberação do diagnóstico.

\section{Análise estatística}

O software criou lotes em bloco de notas digitados em planilha do Microsoft Excel, criando um banco de dados. Para os cálculos da sensibilidade e especificidade foram utilizados a seguintes fórmulas: sensibilidade $=$ verdadeiros positivos/ positivos; Especificidade = verdadeiros negativos/ negativos. Para avaliar as médias de acertos software, foi utilizado o teste de Mann-Whitney. Foi considerado no nível de $5 \%$ de probabilidade em todos os testes e utilizado o Software SSPS 10.0 para as análises.

\section{RESULTADOS}

Desenvolveu-se um software de verificação de imagens retinografia, o qual foi treinado para analise e apoio na realização de diagnósticos. O layout e funcionalidade do programa são expostas na Figura $1 \mathrm{~A}, \mathrm{~B}, \mathrm{C}$.

Para a validação foi utilizado 48 retinografia normais; 140 de descolamento de retina; 53 papiledemas; 255 retinopatias diabéticas; 39 glaucomas; 202 toxoplasmose oculares. No total foram utilizado 161 imagens normais no banco de dados; 500 de deslocamentos; 212 papiledemas; 867 retinopatias diabéticas; 126 glaucomas e 698 toxoplasmose (Tabela 1 e Figura 2).

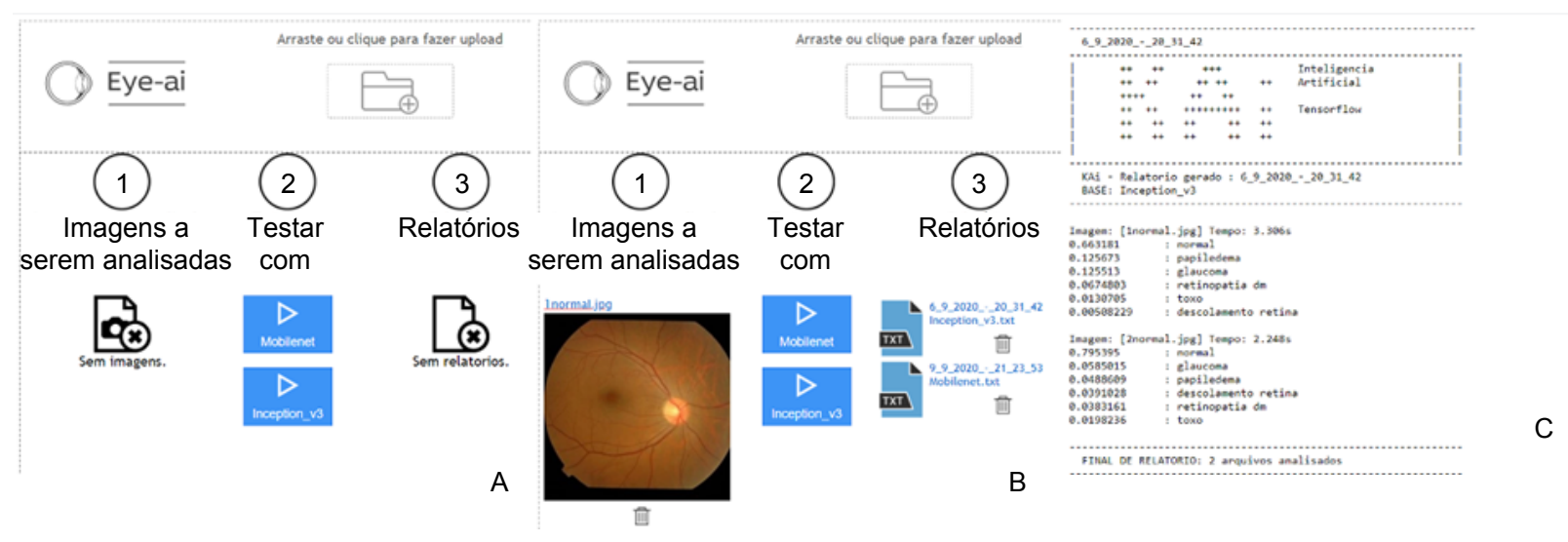

Figura 1: Layout do programa desenvolvido. A: Tela inicial do programa; B: Inserção de fotos para a análise; C:Relatórios desenvolvidos pelo programa. 
Kuiava et al.

Tabela 1: Variáveis utilizadas para a construção do banco de dados do programa.

\begin{tabular}{lcccc}
\hline & Retinografia & Banco de dados & Teste & Total \\
\hline Normal & 48 & 161 & 31 & 192 \\
Descolamento Retina & 140 & 500 & 60 & 560 \\
Papiledema & 53 & 212 & 44 & 212 \\
Retinopatia DM & 255 & 867 & 153 & 1020 \\
Glaucoma & 39 & 126 & 30 & 156 \\
Toxoplasmose & 202 & 698 & 110 & 808 \\
\hline
\end{tabular}

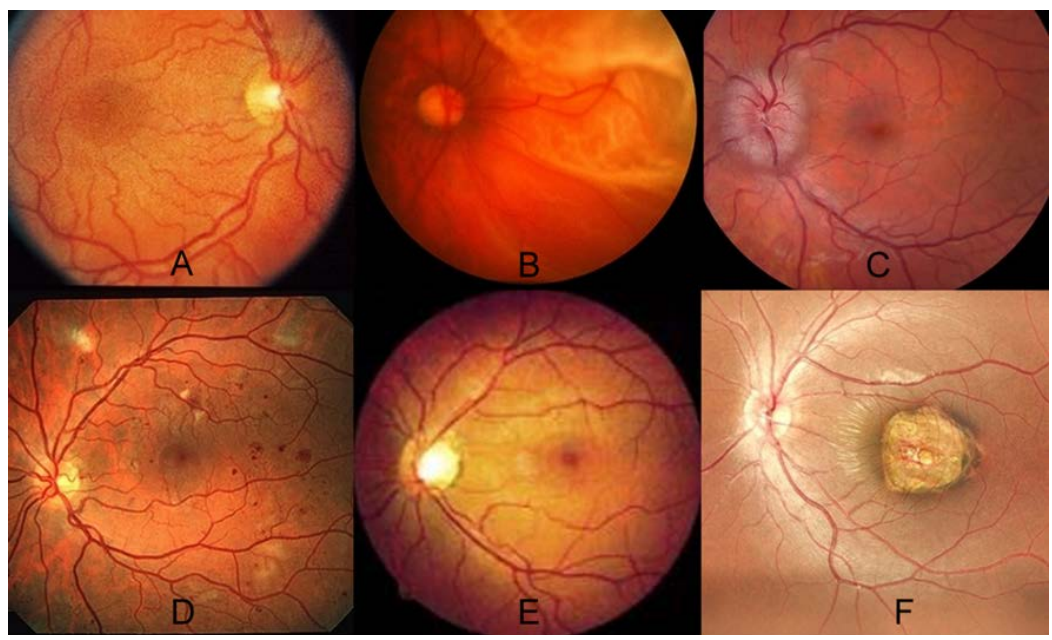

Figura 2: Acervo de fotos retinianas utilizadas para a construção do programa. A: Retina normal; B: Descolamento de retina; C: Papiledema; D: Retinopatia diabética; E: Glaucoma; F: Toxoplasmose retiniana.

Foi calculado a sensibilidade e especificidade para cada um dos modelos computacionais usados. No MobileNet, obteve-se uma sensibilidade total de $91 \%$ (IC 95\%, 89-92\%) e uma especificidade de $98,5 \%$ (IC 95\%, 98-99\%). No Inception, foi de
91,4\% (IC 95\%, 89-93,5\%) de sensibilidade e 98,4\% (IC 95\%, 98-98,8\%) de especificidade (Tabela 2). Não houve diferenças estatísticas entre os dois modelos desenvolvidos $p=0,83$ na sensibilidade $e$ $p=0,93$ na especificidade.

Tabela 2: Porcentagem de sensibilidade e especificidade de acordo com a alterações patológica.

\begin{tabular}{llcc}
\hline & & MOBILE & INCEPTION \\
\hline \multirow{2}{*}{ Normal } & SENSIBILIDADE & $90,32 \%$ & $87,10 \%$ \\
& ESPECIFICIDADE & $98,11 \%$ & $98,82 \%$ \\
\hline \multirow{2}{*}{ Descolamento Retina } & SENSIBILIDADE & $91,67 \%$ & $93,33 \%$ \\
& ESPECIFICIDADE & $99,29 \%$ & $98,58 \%$ \\
\cline { 2 - 4 } Papiledema & SENSIBILIDADE & $86,36 \%$ & $90,91 \%$ \\
& ESPECIFICIDADE & $97,88 \%$ & $98,58 \%$ \\
\hline \multirow{2}{*}{ Retinopatia DM } & SENSIBILIDADE & $91,28 \%$ & $90,60 \%$ \\
\hline \multirow{2}{*}{ Glaucoma } & ESPECIFICIDADE & $98,58 \%$ & $97,41 \%$ \\
\hline \multirow{2}{*}{ Toxoplasmose } & SENSIBILIDADE & $93,33 \%$ & $96,55 \%$ \\
& ESPECIFICIDADE & $99,29 \%$ & $99,06 \%$ \\
\hline \multirow{2}{*}{ Global } & SENSIBILIDADE & $93,64 \%$ & $90,00 \%$ \\
& ESPECIFICIDADE & $98,35 \%$ & $98,35 \%$ \\
\hline
\end{tabular}




\section{DISCUSSÃO}

Dados da Organização Mundial da Saúde (OMS) e que constam no recente documento "As Condições da Saúde Ocular no Brasil 2019", elaborado pelo Conselho Brasileiro de Oftalmologia (CBO), mostram que atualmente, estima-se que a cegueira afeta 39 milhões de pessoas em todo o mundo e que 246 milhões sofram de perda moderada ou severa da visão ${ }^{15}$. Desta forma, os processos de detecção precoce e correto diagnóstico de alterações oftalmológicas dependem, dentre outros fatores, do raciocínio e experiência de oftalmologistas. O uso de técnicas computacionais com diagnóstico estruturado em IA torna-se uma ferramenta importante que poderá ajudar oftalmologistas e especialmente os pacientes, melhorando e acelerando o diagnóstico desta alterações. Este trabalho propôs o desenvolvimento de um software que poderá auxiliar no diagnóstico das patologias oftalmológicas mais relevantes.

Os resultados obtidos mostraram que o Software EYE gerou dados promissores, apresentando sensibilidade de $91 \%$ e especificidade de $98 \%$, com características inovadoras que poderiam ser incorporadas no futuro em clínicas, hospitais, consultórios, unidades básicas de saúde, contribuindo para um diagnóstico oftalmológico mais rápido e efetivo. O sistema apresentou resultados promissores para o diagnóstico de todas as patologias analisadas, deslocamento de retina, papiledema, retinopatia diabética, glaucoma, toxoplasmose, bem como, da diferenciação entre normalidade e patologias.

Programas computacionais que utilizam inteligência artificial tem se mostrado com grande capacidade de auxiliar em áreas da saúde. Os resultados são promissores com elevada sensibilidade e especificidade, apresentando-se como ferramentas potenciais para diagnostico destas enfermidades, podendo tornar-se forma complementar à assistência integral do paciente ${ }^{6}$. Alguns estudos têm demonstrado que sistemas de IA podem até superar os profissionais no diagnóstico de enfermidades específicas. Um estudo publicado em Annals of Oncology Scientific Journal, por Holger Haenssler, professor no departamento de dermatologia da Universidade de Heidelberg, na Alemanha, com participação de cientistas da França e Estados Unidos, mostrou que patologistas detectaram com precisão $86,6 \%$ de melanomas, enquanto que um sistema de IA obteve acerto em $95 \%$ dos $\operatorname{casos}^{16}$. Bringsjord et al descreveram que na área da saúde, o uso de sistemas de IA tem apresentado significativos progressos, sendo assim visto como uma das principais formas de auxiliar diagnósticos ${ }^{17,18}$.

Sistemas de apoio à decisão propõem ajudar os clínicos no diagnóstico e decisões terapêuticas relacionadas ao paciente. Eles também facilitam o acesso dos profissionais a dados precisos na literatura científica, possibilidades propedêuticas, estabelecimento de diagnóstico diferencial, além de destacar características específicas em novos padrões clínicos ${ }^{19}$. Essa tecnologia pode auxiliar em muitos países como o Brasil que apresenta dimensões continentais, onde ainda há má distribuição de algumas especialidades médicas, dentre ela a oftalmologia ${ }^{20}$. A agregação de tecnologia na assistência aos pacientes contribui para o diagnóstico e eventual conduta mesmo em áreas geográficas que não possuem especialistas ${ }^{21}$.

O sistema de leitura e identificação facilita a leitura e diagnóstico de imagens oftalmológicas, permitindo que oftalmologistas possam diagnosticar as mesmas estruturas à distância, como em cidades afastadas de centros metropolitanos, vilas e regiões ribeirinhas que não contam com profissionais capacitados para o diagnóstico ${ }^{22,23}$. Esse tipo de procedimento tem potencial de reduzir vários custos e também garantir a realização de um diagnóstico mais rápido e assertivo ${ }^{24}$. Com base no banco de dados, o sistema de leitura e diagnóstico é capaz de disponibilizar recomendações mais enfáticas para cada patologia em alguns minutos, mostrando também o melhor tratamento.

As características que conferem potencial de uso médico no programa aqui apresentado são a grande utilização de imagens da retina e do nervo óptico para a construção do banco de dados e na interface intuitiva para a avaliação do programa. O EYE diferencia-se por possuir dois bancos de dados pré-treinados, que potencializa a validação dos dados, garante maior segurança de resultados e melhor validação externa e pode ser incluído como medida complementar no programa de governo da Política Nacional de Atenção em Oftalmologia, n. ${ }^{\circ} 957$, de 16 de maio de 2008 , através da articulação de assistência entre os sistemas de cuidado dos pacientes. Exemplos dessa integração já foram realizados por parcerias de público-privado na Índia como forma de maximizar o fluxo de paciente para o atendimento especializado da retinopatia diabética ${ }^{14}$.

Destacamos o fato de que é improvável que uma máquina substitua inteiramente profissionais humanos ${ }^{25,26}$. A Assistência de profissionais de saúde sempre será fundamental na área de cuidado ao paciente ${ }^{27,28}$. A necessidade de avaliação integral das características clínicas e individuais, torna improvável a completa substituição de toda equipe de saúde pela tecnologia. À medida que esta é incorporada à avaliação médica, torna-se necessário atualização especializada para domínio destas novas técnicas ${ }^{10}$.

Programas computacionais que utilizam inteligência artificial apresentam grande potencial para melhorar a assistência médica aos pacientes. 
O programa desenvolvido apresentou elevados valores de sensibilidade e especificidade, 91 e $98 \%$, respectivamente. A incorporação de programas de inteligência artificial como apoio ao profissionais de saúde, junto com a tecnologia já existente a serviço da medicina pode contribuir de forma significativa na eficiência diagnóstica e avaliações médicas a distância e em locais onde o especialista não é disponibilizado

\section{Conflitos de interesse}

Os autores negam qualquer conflito de interesse

\section{REFERÊNCIAS}

1. Souza AR, Talon AF. Inteligência Artificial Aplicada à Medicina. Cad Estud Tecnológicos. 2013;1(1):14.

2. Szolovits P. Artificial intelligence and medicine. Artif Intell Med. 2019;41(2):1-19.

3. Dutt S, Sivaraman A, Savoy F, Rajalakshmi R. Insights into the growing popularity of artificial intelligence in ophthalmology. Indian $\mathrm{J}$ Ophthalmol. 2020;68(7):1339.

4. Moraru A, Costin D, Moraru R, Branisteanu D. Artificial intelligence and deep learning in ophthalmology present and future (Review). Exp Ther Med. 2020;20:3469-73.

5. Chielle EO, Kuiava EL, Kuiava VA. Sistema estruturado com inteligência artificial para apoio no diagnóstico de parasitas intestinais. Anais do $26^{\circ}$ Seminário de Iniciação Científica e do $13^{\circ}$ Seminário Integrado de Ensino, Pesquisa e Extensão da Unoesc;2020 Oct 19-23; Joaçaba, SC. Joaçaba: Editora da Unoesc; 2020.

6. Kuiava VA, Kuiava EL, Rodriguez R, Beck AE, Rodriguez JPM, Chielle EO. Method of histopathological diagnosis of mammary nodules through deep learning algorithm. J Bras Patol e Med Lab. 2019;55(6):620-5.

7. Santos MK, Wada DT, Priscilla A, Henrique M, Barbosa N, Mazzoncini P, et al. Inteligência artificial, aprendizado de máquina, diagnóstico auxiliado por computador e radiômica : avanços da imagem rumo à medicina de precisão. Radiol Bras. 2019;52(6):387-96.

8. Ting DSW, Lin H, Ruamviboonsuk P, Wong TY, Sim DA. Artificial intelligence, the internet of things, and virtual clinics: ophthalmology at the digital translation forefront. Lancet Digit Health. 2020;2(1):e8-9.

9. Korot E, Wood E, Weiner A, Sim DA, Trese M. A renaissance of teleophthalmology through artificial intelligence. Eye (Lond). 2019;33(6):861-3.
10. Padhy SK, Takkar B, Chawla R, Kumar A. Artificial intelligence in diabetic retinopathy: A natural step to the future Srikanta. Indian J Ophthalmol. 2019;67(7):1004.

11. RedCheck [Internet]. São Paulo: RedCheck; 2020 [cited 2020 Nov 12]. Available from: https://redcheck.com.br/site

12. Kara José AC, Passos LB, Kara José FC, Kara José N. Ensino extracurricular em Oftalmologia: grupos de estudos/ligas de alunos de graduação. Rev Bras Educ Med. 2007;31(2):166-72.

13. Carvalho JA. Oftalmologia e realidade virtual. Rev Bras Oftalmol. 2012;71(1):40-7.

14. Brasil. Portaria $n^{\circ} 957$, de 15 de maio de 2008. Diário Oficial da União. 2008 May 16;1:43.

15. Ottaiano JAA, Ávila MP, Umbelino CC, Alexandre T. As Condições de Saúde Ocular no Brasil. São Paulo: Conselho Brasileiro de Oftalmologia; 2019.

16. Mar VJ, Soyer HP. Artificial intelligence for melanoma diagnosis: How can we deliver on the promise? Ann Oncol. 2018;29(8):1625-8.

17. Stone P, Brooks R, Brynjolfsson E. Artificial intelligence and life in 2030. Stanford: Stanford University; 2016.

18. Bringsjord S, Bello P, Ferrucci D. Creativity, the Turing Test, and the (Better) Lovelace Test. Minds Machin. 2001; 11:3-27.

19. Brynjolfsson E, McAfee A. The Second Machine Age. New York: WW Norton Co.; 2014.

20. Nogueira PTA, Bezerra AFB, Leite AFB, Carvalho IMDS, Gonçalves RF, de Brito-Silva KS. Características da distribuição de profissionais do Programa Mais Médicos nos estados do Nordeste, Brasil. Cienc Saude Colet. 2016;21(9):2889-98.
21. Cossy-Gantner A, Germann S, Schwalbe NR, Wahl B. Artificial intelligence (AI) and global health: How can Al contribute to health in resource-poor settings? BMJ Glob Heal. 2018;3(4):1-7.

22. Krizhevsky A, Sutskever I, Hinton GE. ImageNet Classification with Deep Convolutional Neural Networks. Proceedings of the 25th International Conference on Neural Information Processing Systems; December 3-6, 2012; Lake Tahoe, NV. Red Hook: Curran Associates; 2012.

23. Gunkel DJ. Comunicação e inteligência artificial: novos desafios e oportunidades para a pesquisa em comunicação. Galaxia. 2017;34(2012):5-19.

24. Atun R. Transitioning health systems for multimorbidity. Lancet. 2015;6736(14):8-9.

25. Badawi O, Brennan T, Celi LA, Feng M, Ghassemi M, Ippolito A, et al. Making Big Data Useful for Health Care: A Summary of the Inaugural MIT Critical Data Conference. JMIR Med Inf. 2014;2(2):22.

26. Sugi M, Murakami H, Yoshida K. Projection of future changes in the frequency of intense tropical cyclones. Clim Dyn. 2017;49(1-2):619-32.

27. Kuiava VA, Pavan NR, Grisolia ET, Hoppe L, Reichert PR, Navarini D. Ecological study of viral hepatitis in Brazil: A geographical and temporal analysis. Clin Biomed Res. 2019;39(2):122-7.

28. Kuiava VA, Chielle EO. Epidemiology of cervix cancer in Brazil (20052015): study of mortality and hospital intervention rates. Arch Biosci Heal. 2019;1(1):45-60. 